\title{
Regimewechsel der Abfallpolitik
}

\section{Prozesse der europäischen Harmonisierung starten von unterschiedlichen} nationalen Regimen aus und werden von deren Besonderheiten geprägt. Die Regime werden wiederrum wesentlich durch Dynamiken von "innen" und von "außen" beeinflußt. Dies zeigt die Entwicklung der Hausmüllpolitik. In einer vergleichenden Analyse der fünf EU-Staaten Deutschland, Frankreich, Italien, Niederlande und Griechenland werden die Entwicklungspfade der nationalen Regime, die ihnen zugrundeliegenden Konventionen und ihre Interaktion in den letzten 25 Jahren analysiert.

$\mathrm{A}$ Wim Hafkamp bfallregime enstehen nicht über einen kurzen Zeitraum hinweg und nicht (allein) durch politisches Handeln, sondern in einem langen Prozeß und durch ,institution building“. Derzeit befinden sich die Abfallmanagementregime vieler europäischer Länder mitten in einem Wechsel von einem alten Regime, das sich zwischen der Mitte des 19. Jahrhunderts und den 70er Jahren dieses Jahrhunderts etablieren konnte, zu einem neuen Regime, das quer durch Europa am Entstehen ist und sich im nächsten Jahrhundert durchsetzen wird.

Eine einfache Darstellung der Grundprinzipien des alten und des sich entwickelnden neuen Regimes erfolgt in Tabelle 1. Die folgenden Abschnitte geben einen Überblick über die wesentlichen Elemente dieses Regimewechsels. Sie haben zum Ziel, die Ausbalancierung der neuen Wertvorstellungen nachzuzeichnen, und zu erklären, wie diese die Prinzipien der Konventionen, die den Abfallregimes zugrundeliegen, beeinflußt haben. Gleichzeitig wird gezeigt, wie der Gegenstand der Konventionen, also Politiken, institutionelle Ausgestaltung und Technologien als Bestandteile der Regime, durch die neuen Wertvorstellungen beeinflußt wird.

\section{Stillstand bis in die späten $70 \mathrm{er}$}

Noch Anfang bis Mitte der 70er Jahre erweist sich das alte System der Abfallwirtschaft als gefestigt. Die Überflußgesellschaft, die sich in Europa und den anderen westlichen Industriestaaten ausbreitet, führt zu Umweltproblemen, aber zugleich auch zu einer zunehmenden Wahrnehmung dieser Probleme. Der Bericht von Meadows an den Club of Rome ,Grenzen des Wachstums“ stellt eine Verbindung her zwi- schen der Verknappung der Ressourcen sowie dem Abfallproblem und der Umweltverschmutzung. Die Umweltbewegung entwickelt sich und Materialismus und Konsumgesellschaft geraten in die Kritik. Die Umwelt wird zum Kern einer Wertediskussion. Umweltgruppen entwickeln, zunächst in den USA, ein neues Leitprinzip für die Abfallpolitik: die ,prevention ladder" (Vorsorgehierarchie):

Aus diesem Prinzip entwickelte sich ein neuer Typus von Umweltpolitik, der sich als ,Umwelthygiene" bezeichnen läßt, vor allem auf Umweltverschmutzung ausgerichtet war und den Schwerpunkt auf Luft-, Wasser- und Bodenqualität legte. Diese umweltpolitischen Vorstellun- gen wurden auch auf das Abfallmanagement übertragen. Dabei wurden zwei Elemente hinzugefïgt:

- Den regionalen Behörden (Länder oder Provinzen) wurde die Zuständigkeit übertragen, die Abfallplanungen zu koordinieren.

- Es wurde eine Genehmigungspflicht für Abfallbehandlungsanlagen eingeführt, die der für Industrieanlagen sehr ähnelt.

Die Abfallgesetzgebung wurde in Deutschland 1975 und in Holland 1978 eingeführt. Diese Gesetze bestätigten im Grundsatz, daß das Management von Haushaltsabfällen eine öffentliche Aufgabe ist.

Die vorherrschende Technologie der Abfallbehandlung blieb - die vielfach unkontrollierte Deponierung. Allerdings spielen die natürlichen Bedingungen eine Rolle. In Italien wurden zum Beispiel Steinbrïche als Deponien benutzt. In dicht besiedelten Regionen entwickelte sich die Verbrennung als zweite wichtige Technologie der Müllbeseitigung. Mit der Zunahme der Abfallströme und den strenger werdenden Umweltstandards nahm die Bedeutung von Großanlagen zu. Unter dem Druck der Umweltbewegung vor allem in Deutschland, Dänemark und den Niederlanden begann in den 70er Jahren ein politischer Prozeß, der zum Ziel hatte, die ,prevention ladder" durchzusetzen. Die Zielscheibe der Aktionen der Umweltbewegung waren Einwegverpackungen als Symbol der ,Wegwerfgesell-

\begin{tabular}{|c|c|c|}
\hline & Altes Regime & Neves Regime \\
\hline Entstehung & Seit Mitte des 19. Jahrhunderts & Seit Mitte der 70er Jahre \\
\hline Zentrole Wertvorstellungen & Öffentliche Gesundheit & $\begin{array}{l}\text { Verantwortliches Management } \\
\text { der natürlichen Ressourcen }\end{array}$ \\
\hline Gründe & $\begin{array}{l}\text { Abfall als sanitäres Problem - } \\
\text { Gesundheitsvorsorge }\end{array}$ & $\begin{array}{l}\text { Kontrolle des physischen Meta- } \\
\text { bolismus der Gesellschaft }\end{array}$ \\
\hline Konventionen & $\begin{array}{l}\text { Die Gemeinde ist zustöndig für regelmäBige } \\
\text { Abfallsammlung und -tronsport. } \\
\text { Die Entsorgung wird ohne Gefährdung der } \\
\text { öffentlichen Gesundheit gesichert. } \\
\text { Der Nutzer zahit: } \\
\text { Houshalte unterliegen dem AnschluBzwang. } \\
\text { Die Gemeinde ist verpflichtet } \\
\text { alle Abfälle anzunehmen. }\end{array}$ & $\begin{array}{l}\text { Leitbild: „prevention ladder" } \\
\text { (Vermeidung vor Verwertung } \\
\text { vor Beseitigung) } \\
\text { - Schliefung der Kreislöufe } \\
\text { - Varsichtsprinzip } \\
\text { Erweiterte } \\
\text { Produktverantwortung }\end{array}$ \\
\hline Räumliche Reichweite & Lokal & Von global bis lokal \\
\hline Vorherrschende Technologien & $\begin{array}{l}\text { Deponierung } \\
\text { Verbrennung }\end{array}$ & $\begin{array}{l}\text { Vermeidungsansätze } \\
\text { Wiederverwendung, } \\
\text { Recycling, Verwertung }\end{array}$ \\
\hline Institutionelle Regelungen & $\begin{array}{l}\text { - Gemeinden sind zuständig } \\
\text { (entsorgungspflichtig). } \\
\text { - Übergeordnete Instanzen } \\
\text { koordinieren und regulieren. }\end{array}$ & $\begin{array}{l}\text { In der Entwicklung begriffen } \\
\text { Verantwortungsverlagerung auf } \\
\text { die Industrie, dort auch } \\
\text { praktische Durchführung }\end{array}$ \\
\hline
\end{tabular}


schaft". In Deutschland und auch den Niederlanden hatte dies allerdings zunächst wenig Einfluß auf das Abfallregime. Diese erwiesen sich überall in Europa als sehr stabil und anpassungsfähig an die neuen Anforderungen des Umweltschutzes.

\section{- Anfang bis Mitte der 80 er: die Abfallregime geraten unter Druck}

In den frühen 80er Jahren geraten durch zwei Faktoren die Haushaltsabfallregime unter Druck:

Der sich im Geleit mit der Umweltpolitik in den 70er Jahren entwickelnde Veränderungsprozeß beschleunigte sich.

- Zusätzlich gab es in Verbindung mit der Thatcher/Reagan-Ära eine Privatisierungswelle von bislang öffentlich wahrgenommenen Aufgaben, die auch den Abfallbereich betraf.

Die frühen 80er waren in vielen europäischen Staaten gekennzeichnet durch eine Reihe von Unfällen auf Abfalldeponien mit der Folge von Boden- und Grundwasserkontaminationen. Das NIMBY-Syndrom (,Not In My Backyard“) trat nun auch in der Abfallpolitik in Erscheinung. Es wurde immer schwieriger, neue Flächen selbst für kontrollierte Abfalldeponien zu finden. In zeitlich kurzem Abstand entwickelte sich ein ähnlicher Prozeß aufgrund der Dioxin-Emissionen auch bei Abfallverbrennungsanlagen. Das Resultat beider Prozesse waren

1. strengere Umweltstandards,

2. ein erheblicher Anstieg der Kosten und

3. eine Zunahme der Größe der Anlagen, um die steigenden Kosten in den Griff zu bekommen.

Das zunehmende Volumen der Haushaltsabfälle wurde zudem zu einem wesentlichen endogenen Faktor. Die Deponiekapazitäten wurden knapp. In der Folge wurden in allen hier betrachteten Ländern mit Ausnahme von Griechenland Abfallpolitiken entwickelt. Für Frankreich und Italien stellte dies die ,erste Runde“ dar, die in Italien aber nur auf dem Papier stattfand. In Holland und Deutschland wurde 1988 bzw. 1989 die ,prevention ladder" als Orientierungsgröße für die Abfallpolitik genutzt. Daraus folgten neue Initiativen hinsichtlich getrennter Sammlung und Recycling, und erste Ansätze einer auf Verpackungen ausgerichteten Politik.

Was Wertvorstellungen und Prinzipien betrifft, kann ein Prozeß wahrgenommen werden, in welchem Umwelt"values" sich stärker durchsetzen konnten. Dies löste eine Diskussion aus, wie ökologische Effektivität und ökonomische Effizi- enz gemeinsam erreicht werden könnten. Aber auch bis zu diesem Zeitpunkt hat kein grundlegender Wandel der Konventionen und der ihnen zugrundeliegenden Prinzipien stattgefunden. Obwohl die ,prevention ladder" bereits deutlich früher übernommen wurde, hatte sie erst jetzt Auswirkungen auf die Abfallpolitik, da die nationalen Politiken in einigen Ländern verstärkte Bemühungen an den Tag legten, um das Recycling und die Verbrennung mit Energierïckgewinnung zu forcieren.

Ein wesentlicher Wandel läßt sich jedoch feststellen: mit dem Eingang der ,prevention ladder" in die Abfallpolitik kam ein neues Prinzip zu dem Regime hinzu, das eine neue Orientierung gab: Von „Umwelthygiene bei der Abfallverbringung“ zum „Management von Materialien und Stoffflüssen". Die erstgenannte Ausrichtung hatte keinen tiefgreifenden Einfluß auf das Regime, da sie letztlich durch End-of-pipe-Lösungen in den Griff bekommen werden konnte. Dies trifft für den zweiten Fall nicht mehr zu.

Verschiedene „Bruchstellen“ der Regime wurden nunmehr sichtbar, die im Verlauf der Entwicklung erhebliche Auswirkungen gehabt haben:

1. Die räumliche und administrative Ebene der Verantwortung der entsorgungspflichtigen Körperschaften im Hinblick auf die (meist kommunale) Sammlung und die Ebene der Behandlung von Abfällen (regional, national) paßten immer weniger zusammen.

2. Es entstand zum ersten Mal ein Wettbewerb zwischen unterschiedlichen Abfallbehandlungsmethoden (Deponierung, Verbrennung, Recycling); was insbesondere deshalb zum Problem wurde, da eine Reihe von entsorgungspflichtigen Körperschaften hohe Investitionen in großdimensionierte Beseitigungsanlagen getätigt hatten, die zugleich langfristige finanzielle Verpflichtungen bedeuteten. Damit entstand eine neue Form des Wettbewerbs zwischen öffentlichen Gebietskörperschaften.

3. Die privaten Unternehmen spielen eine zunehmende Rolle bei der separaten Sammlung und dem Recycling oder der Wiederverwendung von Haushaltsabfällen. Der Wettbewerb öffentlich - privat wurde ebenfalls zu einer treibenden Kraft.

Der zweite wesentliche Trend Mitte der achtziger Jahre ist Privatisierung und Deregulierung. Während z.B. in Italien die private Sammlung von Haushaltsabfällen bereits seit langem üblich war, hat dieser Trend in einigen Ländern wie Deutsch- land, Frankreich und Holland dazu gefiihrt, die Aufgabenteilung staatlich-privat $\mathrm{zu}$ überdenken. Für Frankreich ist in dem Zusammenhang charakteristisch, daß sich frühzeitig starke private Mehrzweckunternehmen wie Vivendi und Lyonnaise des Eaux entwickeln konnten.

All dies fïhrte auch zu einer starken Professionalisierung, bei der nicht allein neue Technologien eingesetzt wurden, sondern zugleich auch neue Logistikkonzepte und neue Organisationsformen einschließlich neuer Unternehmensorganisationen entstanden.

\section{Seit den späten 80ern: Friktio- nen zwischen nationalen Regimen}

Auch in Griechenland wurde jetzt die Notwendigkeit, neue Abfallbehandlungsanlagen zu entwickeln, drängend. Verschiedene Versuche der Behörden scheiterten jedoch am Widerstand der Kommunen und endgültig vor Gericht. Gegenwärtig ist Griechenland dabei, einen Abfallmanagementplan vorzubereiten, der vorsieht, daß keine Anlage mehr in der bisher geplanten Größe erforderlich wird. Statt dessen soll eine Kombination von Anlagen genutzt werden, um Glas zu separieren, zu kompostieren, Brennstoffe aus Müll zu produzieren und um zu recyceln. Dieser Planungsprozeß zeigt, daß ,stage skipping“, also das Überspringen von Entwicklungsstufen, eine realistische Option darstellt, zumindest unter dem Druck zunehmend knapper werdender Deponiekapazität und der absoluten Ablehnung der klassischen Verbrennung durch die Griechen.

Auch das französische Regime ist aufgrund von internen und externen Faktoren in Bewegung gekommen. Die „prevention ladder" wurde angenommen, wenn auch nicht so strikt wie in Deutschland. Es wurde das Konzept der ,Valorisation“ eingefuihrt, das alle Methoden abdeckt mit denen Abfall verwertet werden kann. Die Ratio dabei ist, daß

1. es sehr schwierig, wenn nicht gar unmöglich ist, eine Vorteilhaftigkeit der Wiederverwendung gegenüber dem Recycling und der Verbrennung aus ökologischer Perspektive aufrechtzuerhalten und daß

2. aus Sicht der technologischen Entwicklungsdynamik es auf längere Sicht nicht effektiv ist, Abfallbehandlungstechnologien vorzuschreiben. An dieser Ratio orientierte sich Frankreich auch bei der Diskussion um die Einführung der europäische Verpackungsdirektive Anfang der 90er Jahre und bei ihrer Umsetzung. 
In den Abfallpolitik von Deutschland und den Niederlanden wurde die ,prevention ladder“ zunehmend das handlungsleitende Prinzip. In beiden Ländern wurden 1988/89 umfangreiche Abfallpolitikanalysen mit dem Ziel durchgeführt, Abfallströme zu identifizieren und für diese zielgerichtete Politiken zu entwickeln. Die Mehrzahl der Politiken war auf Produkte orientiert, was implizierte, daß die Industrie (Produzenten oder Importeure) Systeme zu entwickeln hatte, um die Produkte beim Endverbraucher einzusammeln und sie anschließend zu recyceln oder wiederzuverwenden. Damit wurde das neue Prinzip der erweiterten Produktverantwortung eingeführt. Bei unterschiedlichen Produktgruppen (Batterien, Kühlschränke, Fernseher etc.) hatte dies für das Haushaltsabfallregime nur eine untergeordnete Bedeutung: Das Volumen dieser Produkte war eher gering.

Es gibt allerdings eine Ausnahme. In beiden Ländern wurde Verpackungsabfall als ,prioritärer“ Abfallstrom identifiziert, da es sich nach den kompostierbaren Stoffen um die größte Hausmüllfraktion handelte. In der Folge begannen beide Länder mehr oder weniger zeitgleiche politische Initiativen. Die Regierungen beider Länder beabsichtigten, eine Verpackungspolitik zu entwickeln, bei der die Verantwortung auf die Industrie verschoben wurde. In Deutschland fuihrte das 1990, unter dem starken Einfluß der Umweltbewegung, zur Einführung des Dualen Systems DSD.

Bei der skizzierten Divergenz zwischen den nationalen Regimen führte die Dynamik in Richtung einheitlicher Markt und Währung zu steigenden Spannungen zwischen den Regimen. Eine Episode in der dies besonders klar wurde, war der Formulierungs- und Verabschiedungsprozeß der Europäischen Verpackungsdirektive (vgl. den Beitrag von Buclet). Doch auch danach kommen die Spannungen im Interpretations- und (ggf. auch fehlenden) Implementierungsprozeß zum Ausdruck.

Sowohl bezüglich der angewandten Prinzipien als auch der angenommenen Konventionen stellte die deutsche Herangehensweise an das Problem Verpackungsabfall die drastischste Abkehr vom ,,alten“ Hausmüllregime in Europa dar. Sie bewirkte in der Folge, daß diese Verpackungsabfallpolitik sowohl in der EU als auch in den einzelnen Mitgliedsstaaten nachvollzogen wurde. Nachdem sich der Nebel des Politikformulierungsprozesses und der natio- nalen Umsetzung der Verpackungsdirektive gelichtet hat, zeigt sich, daß alle EU-Mitgliedsstaaten gehandelt haben:

1. Sie haben Politiken implementiert und Gesetze verabschiedet, um mit Haushaltsabfällen umzugehen.

2. Sie haben die Verantwortung für den Umgang mit Verpackungsabfällen „upstream“ auf die Produzenten verlagert.

3. Sie haben Organisationen geschaffen, die Gebühren von der Industrie einnehmen, um die Kosten für die getrennte Sammlung (nur in Deutschland) und Recycling (nicht in Holland) zu decken.

Die Länder verwenden allerdings unterschiedliche Definitionen von Recycling. Der französische Ansatz scheint dabei am meisten verbreitet $\mathrm{zu}$ sein. Er wird von mindestens acht EU-Mitgliedsstaaten verfolgt, einschließlich Griechenland und Italien. Dies ist der Fall obgleich die Industrie grundsätzliche Einwände gegen beide dahinterliegenden Prinzipien hat: die ,prevention ladder“" und die erweiterte Produktverantwortung.

\section{Fazit}

Die neunziger Jahre waren weitaus turbulenter als die vorangegangenen Jahre. Die nationalen Haushaltsabfallregime befinden sich in einem Veränderungsprozeß, in dem neue Prinzipien etabliert werden und einige der alten erodieren. Der Veränderungsprozeß startete an verschiedenen Punkten und in verschiedenen Ländern, und benötigte unterschiedlich viel Zeit. Es gibt kein Land, bei dem man das Fazit ziehen könnte, daß sich ein neues Regime bereits etabliert hat. Auf der anderen Seite sind die neuen Prinzipien stark umstritten und eine neue Konvention für den Umgang mit (Nachgebrauchs-)Abfall ist nur partiell und unter großem Widerstand etabliert worden. Unterschiede zwischen nationalen Regimen und Unterschiede in der Geschwindigkeit zwischen den Ländern sind weitgehend durch die Unterschiede in Ausdauer, Stärke und Einfluß von Umweltschutz-NGOs zu erklären. Dies ist vor allem beim ,agenda setting“ von Bedeutung. Umweltschutz-NGOs, die öffentliche Meinung, Verbraucher-NGOs und ,grüne" politische Parteien bilden quasi eine Koalition. Diese Koalitionen sind in Deutschland, den Niederlanden, Skandinavien, Flandern und Österreich wesentlich mächtiger als in Frankreich, Italien, Griechenland oder den anderen EU-Mitgliedsstaaten.

Die Rolle der Industrie ist dabei quer durch Europa hindurch einheitlich, sie unterscheidet sich lediglich in ihrer Effektivität. In Deutschland und in manchen der skandinavischen Länder arbeitet die Industrie mit relativ geringer Effektivität bzw. mit wenig Einfluß auf den politischen Prozeß. Dagegen ist in den Niederlanden aufgrund ihrer geringen Größe und der Konsensorientierung der Einfluß der Industrie größer. In Frankreich sind die relativen Positionen anders: dort gibt es eine kleinere Umweltschutzbewegung mit weniger Einfluß, während der Einfluß der Industrie verhältnismäßig größer und besser organisiert ist. Die Ereignisse im politischen Prozeß der Verabschiedung der EU-Verpackungsdirektive sind dabei ein guter Indikator. In Griechenland und Italien ist der Unterschied im Gewicht von Umweltschutz-NGOs und Industrie noch wesentlich stärker ausgeprägt.

Das alte europäische Abfallregime benötigte eine lange Zeit, um sich zu etablieren und erwies sich als sehr stabil und evolutionär anpassungsfähig. In den 70er und 80er Jahren paßte es sich an das neue Prinzip der „Umwelthygiene“ an, und es kam mit den Privatisierungstrends in den 80er Jahren zurecht. Doch nun hat es erhebliche Schwierigkeiten, sich an die Folgen der ,prevention ladder" und der erweiterten Produktverantwortung, nämlich den Wechsel des Aufgabenbereichs von der Rücknahme des Abfalls und Entsorgung hin zu Stoff- und Materialstrom-Management (in geschlossenen Kreisläufen und langen Kaskaden), anzupassen. Dies wirft natürlich die Frage auf, ob das alte Regime diese Prinzipien zurïckweisen oder zumindest verwässern kann.

Obwohl die zukünftige Richtung unsicher sein mag, scheint es einen gemeinsamen Entwicklungspfad der EU-Mitgliedsstaaten zu geben. Harmonisierung scheint dabei mehr ein Problem des Prozesses (des Erreichens einer Synchronisierung) als ein Problem des Zeitpunktes zu sein.

\section{(Übersetzung: Dirk Hildwein, Uwe Marx)}

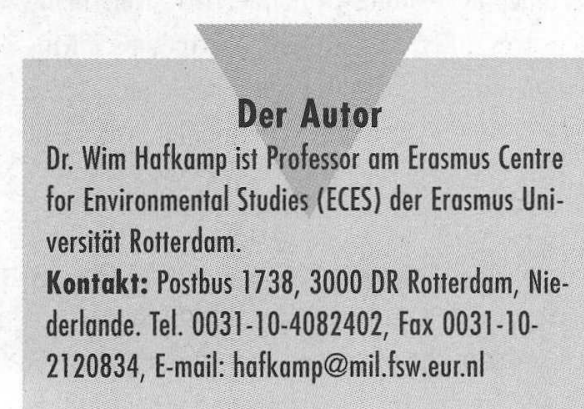


(c) 20I0 Authors; licensee IÖW and oekom verlag. This is an article distributed under the terms of the Creative Commons Attribution Non-Commercial No Derivates License (http://creativecommons.org/licenses/by-nc-nd/3.o/), which permits unrestricted use, distribution, and reproduction in any medium, provided the original work is properly cited. 